\title{
Risk factor modification for the primary and secondary prevention of atrial fibrillation. Part 1
}

\author{
Nebojša Mujović1,2, Milan Marinković ${ }^{1}$, Miroslav Mihajlović², Nataša Mujovićc ${ }^{2,3}$, Tatjana S. Potpara1,3 \\ 1 Cardiology Clinic, Clinical Center of Serbia, Belgrade, Serbia \\ 2 Faculty of Medicine, University of Belgrade, Belgrade, Serbia \\ 3 Clinic for Physical Medicine and Rehabilitation, Clinical Center of Serbia, Belgrade, Serbia
}

\section{KEY WORDS}

atrial fibrillation, lifestyle modification, modifiable risk factors, primary prevention, secondary prevention
Correspondence to: Nebojša Mujović, MD, PhD, Faculty of Medicine University of Belgrade, Dr Subotica 8, 11000 Belgrade, Serbia, phone: +381113616322, email:nmujovic@gmail.com Received: February 24, 2020. Accepted: February 25, 2020. Published online: March 2, 2020. Kardiol Pol. 2020; 78 (3): 181-191 doi:10.33963/KP.15221 Copyright by the Author(s), 2020

\begin{abstract}
Modifiable risk factors, such as cardiometabolic and lifestyle risk factors, considerably contribute to (bi)atrial remodeling, finally resulting in clinical occurrence of atrial fibrillation (AF). Early identification and prompt intervention on these risk factors may delay further progression of atrial arrhythmia substrate and prevent the occurrence of new-onset AF. Moreover, in patients with previous history of recurrent AF, aggressive risk factor management may improve efficacy of other rhythm control strategies, including antiarrhythmic drugs and catheter ablation in sinus rhythm maintenance. Finally, modification of risk factors improves overall health and reduces cardiovascular mortality and morbidity. The first part of this review evaluates the association between AF and the following risk factors: hypertension, diabetes mellitus, physical activity, and cigarette smoking. We systematically discuss the impact of risk factor modification on primary and secondary prevention of AF.
\end{abstract}

Introduction Available rhythm control strategies, including antiarrhythmic drugs and catheter-based or surgical ablation, are associated with modest success and significant adverse effects. ${ }^{1-3}$ Clinical occurrence of AF commonly reflects the presence of advanced and irreversible stage of left atrial disease, thus the primary prevention of AF (ie, prevention of new-onset or incident AF) is of utmost importance. ${ }^{4}$

A growing body of evidence supports early identification and aggressive management of modifiable cardiometabolic and lifestyle risk factors in order to delay progression of arrhythmia substrate and prevent clinical AF, as illustrated in FIGURE 1., 6 Moreover, modification of these risk factors reduces cardiovascular mortality and morbidity. ${ }^{1,4}$

Herein, we discuss the associations of risk factors, such as hypertension, diabetes mellitus (DM), physical activity, cigarette smoking, and AF occurrence. The second part of this review will discuss obesity, obstructive sleep apnea, alcohol use, dyslipidemia and AF. We summarize the studies reporting therapeutic effects of risk factor management on the primary and secondary prevention of AF.
Pathophysiological relationship between risk factors and atrial fibrillation Atrial fibrillation results from interaction of triggers, mostly originating from the pulmonary veins (PVs), left atrial substrate, and autonomic nervous system. ${ }^{1,2}$ Pathophysiological link between modifiable risk factors and AF is presented in FIGURE 2.

Hypertension Hypertension and the risk of atrial fibrillation Systemic hypertension affects approximately $50 \%$ of the general population over 50 years of age and it is the most prevalent risk factor for $\mathrm{AF}^{7-9}$ accounting for more incident $\mathrm{AF}$ cases than any other known risk factor. ${ }^{10} \mathrm{Hy}$ pertension is associated with a 1.4- to 2.1-fold increased risk of new-onset AF. ${ }^{7,8,10}$ Even prehypertension increases the risk of AF. ${ }^{8}$ Among middle-aged prehypertensive men, the 35-year risk of incident $\mathrm{AF}$ was 1.5-fold higher in those with systolic blood pressure (SBP) of 128 to $138 \mathrm{~mm} \mathrm{Hg}$ (vs SBP <128 mm Hg) and 1.79-fold higher in those with diastolic blood pressure (DBP) of $80 \mathrm{~mm} \mathrm{Hg}$ or higher (vs $<80 \mathrm{~mm} \mathrm{Hg}$ ). ${ }^{11}$

A significant increase of new-onset AF risk across increasing blood pressure (BP) categories 

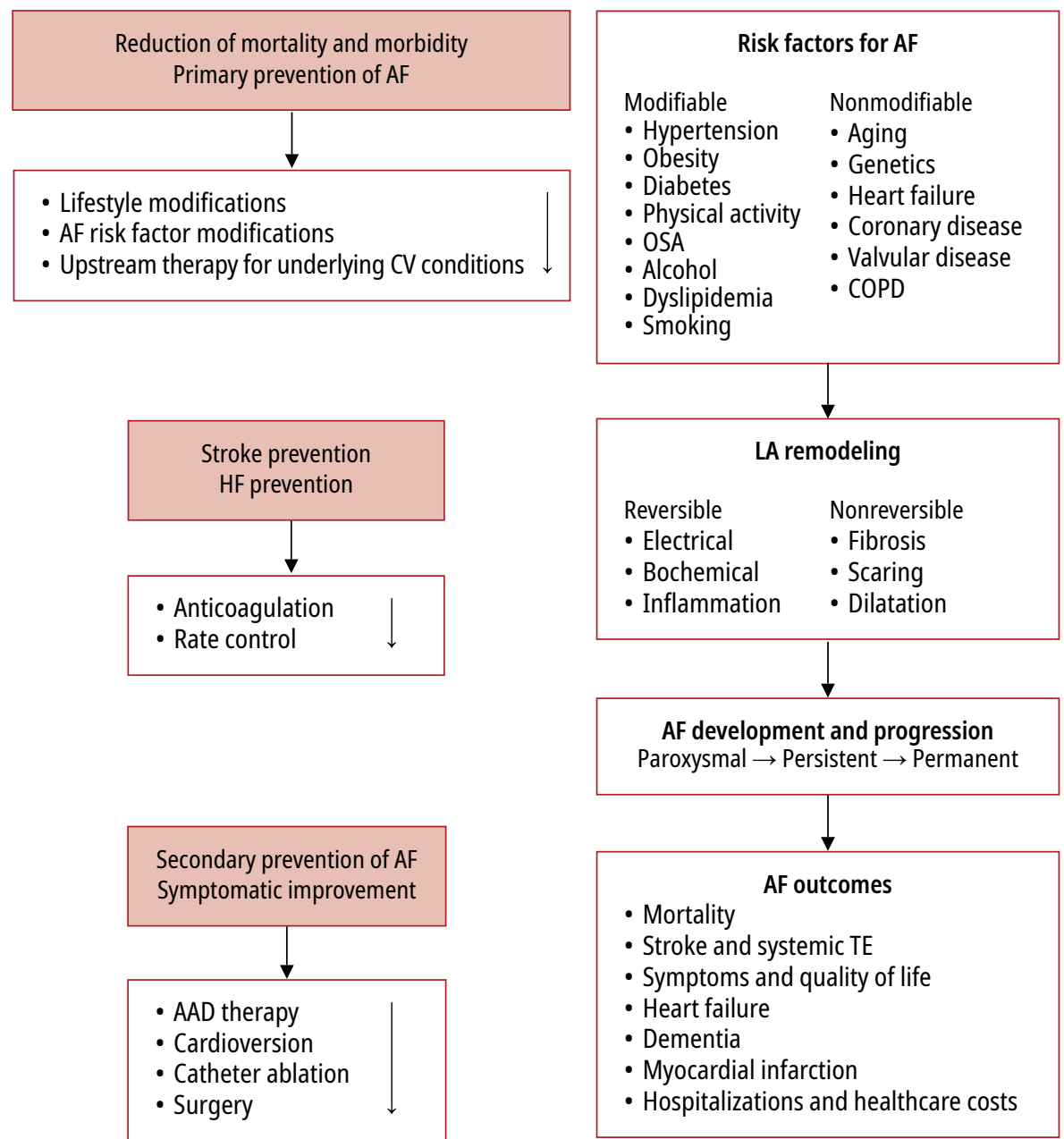

FIGURE 1 Main goals of AF treatment and timelines of different treatment options

Abbreviations: AAD, antiarrhythmic drug; AF, atrial fibrillation; COPD, chronic obstructive pulmonary disease; CV, cardiovascular; $H F$, heart failure; LA, left atrium; OSA, obstructive sleep apnea; TE, thromboembolism

has been reported, ranging from a hazard ratio (HR) of 1.28 for SBP of 120 to $129 \mathrm{~mm} \mathrm{Hg}$ to HR of 2.74 for SBP of $160 \mathrm{~mm} \mathrm{Hg}$ and higher. ${ }^{12}$ A J-shaped association between SBP and risk of incident $\mathrm{AF}$ was demonstrated, with the lowest risk for a SBP of 120 to $130 \mathrm{~mm} \mathrm{Hg}$ and a significant increase in AF incidence for both SBP of less than $120 \mathrm{~mm} \mathrm{Hg}(\mathrm{HR}, 1.99)$ and SBP higher than $150 \mathrm{~mm} \mathrm{Hg}$ (HR, 2.02-2.27). ${ }^{13}$ Diastolic blood pressure is significantly associated with an increased risk of incident AF only at the level of $95 \mathrm{~mm} \mathrm{Hg}$ and higher, suggesting that systolic hypertension is a stronger predictor of new-onset AF than diastolic hypertension. 8,10,12,13

Preprocedural hypertension is a risk factor for AF recurrence after electrical cardioversion in older patients ${ }^{14}$ as well as after catheter ablation in AF (HR 2.5-3.2, with normotensive patients used as the reference). ${ }^{2,15,16}$

Hypertension management and the prevention of atrial fibrillation Primary prevention Lowering SBP to $130 \mathrm{~mm} \mathrm{Hg}$ or less in hypertensive patients with left ventricular (LV) hypertrophy reduces the risk of incident $\mathrm{AF}$ by $40 \%$, while pursuing SBP below $125 \mathrm{~mm}$ Hg provides no additional benefit in the prevention of AF. ${ }^{17}$ The renin-angiotensin-aldosterone system blockers, such as angiotensin-converting-enzyme inhibitors (ACEIs), angiotensin receptor blockers (ARBs), and mineralocorticoid receptor blockers, in comparison with other antihypertensive drugs, yielded some additional, class-specific benefits with respect to the prevention of $A F$ beyond simple $B P$ control, supporting the profound involvement of the renin-angiotensin-aldosterone system in AF pathogenesis. ${ }^{18}$ Monotherapy with ACEIs, ARBs, and $\beta$-blockers in hypertensive patients provided a superior prevention of incident AF compared with calcium channel blockers or diuretics. ${ }^{19,20}$ The ACEIs and ARBs showed the greatest prevention effect on $A F$ in hypertensive patients with LV systolic dysfunction ${ }^{21}$ and addition of $\beta$-blockers and a mineralocorticoid receptor blocker eplerenone (on top of ACEI/ARB therapy) significantly decreased the rate of incident AF by $27 \%$ and $40 \%$, respectively (TABLE 1). 22,23 


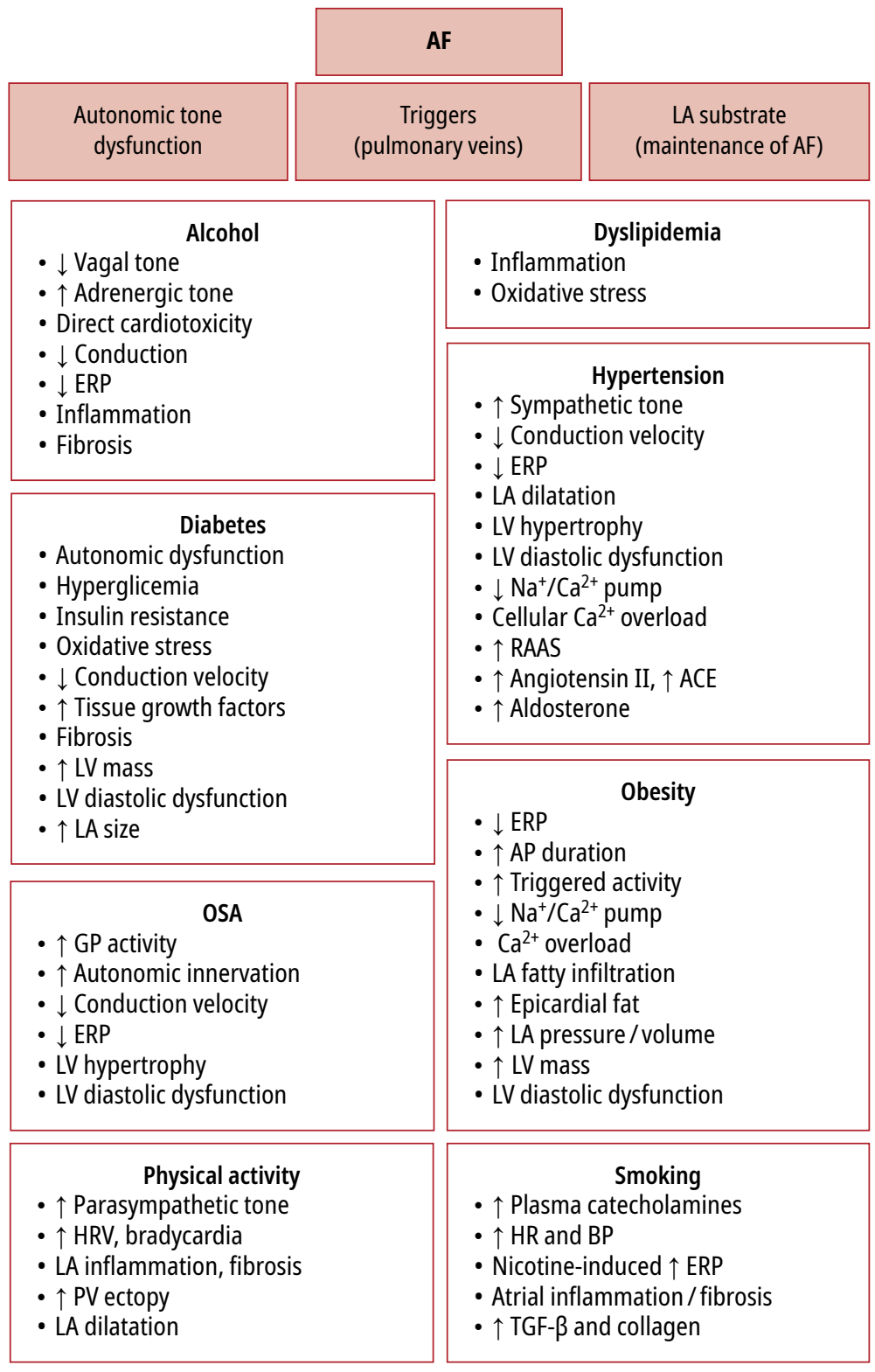

FIGURE 2 Impact of modifiable risk factors on structural and electrical left atrial remodeling predisposing to development of atrial fibrillation

Abbreviations: ACE, angiotensin-converting enzyme; AP, action potential; BP, blood pressure; $\mathrm{Ca}$, calcium; ERP, effective refractory period; GP, ganglionated plexi; HR, heart rate; HRV, heart rate variability; LV, left ventricle; Na, sodium; PV, pulmonary vein; RAAS, renin-angiotensin-aldosterone system; TGF- $\beta$, tumor growth factor beta; others, see FIGURE 1

Secondary prevention Besides their antihypertensive effects, $\beta$-blockers are superior than placebo for the secondary prevention of $\mathrm{AF}^{24}$ and they facilitate a reliable rate control during recurrent AF episodes. ${ }^{1}$ Therefore, $\beta$-blockers are a good choice for hypertensive patients with already documented $\mathrm{AF}$. In patients with $\mathrm{AF}$ with preserved systolic LV function, ACEIs and ARBs were not shown to prevent recurrent $A F$ and they are not recommended for the secondary prevention of paroxysmal AF in patients with no structural heart disease. ${ }^{1}$ Randomized trials failed to demonstrate superiority of valsar$\tan /$ olmesartan over placebo in suppression of recurrent $\mathrm{AF}$ at 1 year. ${ }^{25,26}$ However, $\mathrm{ACEI} / \mathrm{ARBs}$ could be used in patients undergoing cardioversion of persistent $A F,{ }^{1}$ because small randomized trials demonstrated that using enalapril/irbesartan along with amiodarone significantly improved sinus rhythm maintenance post procedure. ${ }^{27,28}$ In patients with resistant hypertension, renal artery denervation performed in conjunction with PV isolation provided a better long-term AF suppression than PV-isolation strategy alone. ${ }^{29}$ The RACE-III (Routine Versus Aggressive Upstream Rhythm Control for Prevention of Early Atrial Fibrillation in Heart Failure) study showed that BP lowering below 120/80 mm Hg improves sinus rhythm maintenance in patients with persistent $\mathrm{AF}$ and heart failure, ${ }^{30}$ whereas the SMAC-AF (Substrate Modification With Aggressive Blood Pressure Control) study reported no benefits of aggressive BP control on postablation rhythm outcome (TABLE 1). ${ }^{31}$

Diabetes mellitus Diabetes mellitus and the risk of atrial fibrillation Patients with DM have a 39\% higher risk of incident AF compared with nondiabetics. ${ }^{32}$ After adjustment for other comorbidities, DM increased the overall risk of new-onset AF significantly more in women (by 26\%) than in men (by $9 \%$ ). ${ }^{33}$ Although younger diabetics had a lower absolute incidence of new-onset AF, their relative risk of $\mathrm{AF}$ was significantly higher than in older patients. ${ }^{34}$ The risk of new-onset $\mathrm{AF}$ increases with duration of DM and worse glycemic control. ${ }^{35}$ Each year of DM and 1-unit increase in hemoglobin $\mathrm{A}_{1 \mathrm{c}}\left(\mathrm{HbA}_{1 \mathrm{c}}\right)$ were associated with a $3 \%$ and $14 \%$ increase in the risk of incident $\mathrm{AF}$, respectively. The risk of incident $\mathrm{AF}$ was considerably higher among patients with DM history of more than 5 years or $\mathrm{HbA}_{1 c}$ level higher than $7 \%$, indicating a threshold relationship between hyperglycemia and AF. ${ }^{35}$ The NAVIGATOR (Nateglinide and Valsartan in Impaired Glucose Tolerance Outcomes Research) trial reported that even in individuals with impaired glucose tolerance (without overt DM), fasting plasma glucose was a predictor of new-onset AF. ${ }^{36}$

Diabetes was an independent risk factor for AF recurrence (odds ratio [OR], 4.6) after electrical cardioversion of persistent AF. ${ }^{37,38} \mathrm{Al}-$ though a long-term AF-freedom after catheter ablation was similar in patients with and without $\mathrm{DM},{ }^{2}$ a higher basal $\mathrm{HbA}_{1 \mathrm{c}}$ level was associated with the risk of late AF recurrence post ablation among patients with DM. ${ }^{39}$ Notably, the quality of glycemic control in the year before catheter ablation of AF was significantly associated with postprocedural AF recurrence within next 12 months. ${ }^{40}$

\section{Treatment of diabetes and the prevention of atrial} fibrillation Primary prevention Studies evaluating the benefits of specific antidiabetic treatment on incident AF reported controversial data. ${ }^{41,42}$ 
TABLE 1 Studies evaluating the impact of antihypertensive therapy on the prevention of atrial fibrillation in hypertensive patients (continued on the next page)

\begin{tabular}{|c|c|c|c|c|c|c|}
\hline Study & Study design & $\begin{array}{l}\text { Study participants } \\
\text { (enrollment criteria) }\end{array}$ & RFM strategy & Control & Follow-up & $\begin{array}{l}\text { Main findings } \\
\text { (AF prevention) }\end{array}$ \\
\hline \multicolumn{7}{|c|}{ Primary prevention of AF } \\
\hline $\begin{array}{l}\text { Okin et al, }{ }^{17} \\
\text { LIFE }\end{array}$ & $\begin{array}{l}\text { Multicenter, } \\
\text { retrospective, } \\
\text { post hoc, } \\
\text { longitudinal }\end{array}$ & $\begin{array}{l}n=8831 \\
\text { Mean (SD) age, } 67(7) \text { y } \\
\text { Essential HTN } \\
\text { ECG criteria of LVH } \\
\text { No previous AF }\end{array}$ & $\begin{array}{l}\text { Atenolol } \\
\text { or losartan for } \\
\text { hypertension }\end{array}$ & None & $\begin{array}{l}\text { Mean } \\
(S D), 4.6 \\
(1.1) \mathrm{y}\end{array}$ & $\begin{array}{l}\text { In patients with LVH, } \\
\mathrm{SBP} \leq 130 \mathrm{~mm} \mathrm{Hg} \\
\text { (vs } \geq 142 \mathrm{~mm} \mathrm{Hg} \text { ) had a } 40 \% \\
\text { risk reduction of incident } \mathrm{AF} \text {. } \\
\mathrm{SBP} \leq 125 \mathrm{~mm} \mathrm{Hg} \text { no longer } \\
\text { associated with the incident } \\
\text { AF risk reduction }\end{array}$ \\
\hline \multirow[t]{2}{*}{$\begin{array}{l}\text { Marott } \\
\text { et al }{ }^{19}\end{array}$} & \multirow{2}{*}{$\begin{array}{l}\text { Retrospective, } \\
\text { observational, } \\
\text { nationwide, } \\
\text { nested, } 1: 1 \\
\text { matched }\end{array}$} & $\begin{array}{l}\mathrm{n}=277880 \\
\text { Median age across } \\
\text { group, } 56-59 \mathrm{y}\end{array}$ & $\begin{array}{l}\text { ACEI vs other } \\
\text { drug class } \\
(n=196092)\end{array}$ & $\begin{array}{l}\beta \text {-Blockers, } \\
\text { CCBs, diuretics, } \\
\text { ARBs }\end{array}$ & \multirow{2}{*}{$\begin{array}{l}\text { Median } \\
\text { across } \\
\text { group, } \\
5.9-6.8 \text { y }\end{array}$} & \multirow{2}{*}{$\begin{array}{l}\text { ACEI or ARBs in patients } \\
\text { without SHD are superior } \\
\text { to } \beta \text {-blockers and diuretics } \\
\text { but not compared with CCBs } \\
\text { in the primary prevention of } \\
\text { AF. }\end{array}$} \\
\hline & & $\begin{array}{l}\text { Monotherapy for HTN } \\
\text { No SHD } \\
\text { No history of AF }\end{array}$ & $\begin{array}{l}\text { ARBs vs other } \\
\text { drug class } \\
(n=81788)\end{array}$ & $\begin{array}{l}\beta \text {-Blockers, } \\
\text { CCBs, diuretics, } \\
\text { ACEI }\end{array}$ & & \\
\hline $\begin{array}{l}\text { Schaer } \\
\text { et } \mathrm{al}^{20}\end{array}$ & $\begin{array}{l}\text { Retrospective, } \\
\text { observational, } \\
\text { nationwide } \\
\text { nested case- } \\
\text {-control }\end{array}$ & $\begin{array}{l}\mathrm{n}=23303 \\
\text { Age range, } 20-79 \text { y } \\
\text { Monotherapy for HTN } \\
\text { No other AF risk factors } \\
\text { No previous AF }\end{array}$ & $\begin{array}{l}\text { Antihypertensive } \\
\text { monotherapy } \\
\text { with ACEI, ARB, } \\
\text { or } \beta \text {-blocker }\end{array}$ & $\begin{array}{l}\text { CCB mono- } \\
\text {-therapy }\end{array}$ & $\geq 1 \mathrm{y}$ & $\begin{array}{l}\text { In patients without SHD, } \\
\text { an ACEI, ARB, and } \beta \text {-blocker, } \\
\text { compared with CCBs, reduces } \\
\text { the risk of new-onset AF } \\
\text { by } 25 \%, 29 \% \text {, and } 22 \% \text {, } \\
\text { respectively. }\end{array}$ \\
\hline \multirow[t]{3}{*}{$\begin{array}{l}\text { Zhang } \\
\text { et al } 21\end{array}$} & \multirow[t]{3}{*}{$\begin{array}{l}\text { Meta-analysis } \\
\text { of } 26 \text { randomized } \\
\text { trials }\end{array}$} & \multirow{3}{*}{$\begin{array}{l}\mathrm{n}=102365 \\
12 \text { trials without AF } \\
\text { history, } 11 \text { trials with } \\
\text { previous AF, } 3 \text { trials with } \\
\text { and without AF } \\
9 \text { trials with HTN } \\
\pm \text { risk factors (CHF, AMI, } \\
\text { DM, or PVD) }\end{array}$} & $\begin{array}{l}\text { ACEIs or ARBs } \\
(n=39405)\end{array}$ & $\begin{array}{l}\text { Placebo or } \\
\text { non-ACEI/ARB } \\
\text { treatment } \\
(\mathrm{n}=41119)\end{array}$ & \multirow[t]{3}{*}{$\begin{array}{l}\text { Mean } \\
\text { across } \\
\text { studies, } \\
6 \text { mo to } \\
6.1 \mathrm{y}\end{array}$} & \multirow{3}{*}{$\begin{array}{l}\text { ACEIs / ARBs had a lower risk } \\
\text { of AF than non-ACEI / ARB } \\
\text { therapy. } \\
\text { ACEIs and ARBs showed } \\
\text { a similar preventive effect } \\
\text { on AF occurrence. } \\
\text { ACEIs / ARBs are better in } \\
\text { secondary (OR, } 0.45) \text { than the } \\
\text { primary prevention of AF } \\
(O R, 0.8) \text {. } \\
\text { More prevention of AF } \\
\text { if EF <40\% }\end{array}$} \\
\hline & & & $\begin{array}{l}\text { ACEIs } \\
(n=10938)\end{array}$ & ARBs $(n=10903)$ & & \\
\hline & & & & & & \\
\hline \multirow[t]{4}{*}{ Nasr et al22 } & \multirow[t]{4}{*}{$\begin{array}{l}\text { Meta-analysis } \\
\text { of } 7 \text { randomized } \\
\text { placebo- } \\
\text {-controlled trials }\end{array}$} & $\begin{array}{l}n=11952 \\
\text { Mean age across group, } \\
57-76 \text { y }\end{array}$ & $\beta$-blocker & Placebo & \multirow[t]{4}{*}{$\begin{array}{l}\text { Mean, } \\
1.35 y\end{array}$} & \multirow{4}{*}{$\begin{array}{l}\text { Addition of a } \beta \text {-blocker } \\
\text { (vs placebo) to ACEI / ARB } \\
\text { therapy is associated with a } \\
27 \% \text { relative risk reduction } \\
\text { in patients with systolic } \\
\text { (but not diastolic) HF. }\end{array}$} \\
\hline & & $\begin{array}{l}\text { Systolic HF } \\
\text { (EF 20\%-36\%) }\end{array}$ & & & & \\
\hline & & ACEIs / ARBs & & & & \\
\hline & & No history of AF & & & & \\
\hline \multirow[t]{3}{*}{$\begin{array}{l}\text { Swedberg } \\
\text { et al, } 23 \\
\text { EMPHASIS- } \\
\text {-HF }\end{array}$} & \multirow[t]{3}{*}{$\begin{array}{l}\text { Subanalysis } \\
\text { of multicenter, } \\
\text { randomized } \\
\text { placebo } \\
\text { controlled trial }\end{array}$} & $\begin{array}{l}n=1794 \\
\text { Age } \geq 55 \text { y } \\
\text { NYHA II class HF } \\
\text { Systolic HF, EF } \leq 35 \%\end{array}$ & $\begin{array}{l}\text { Eplerenone } \\
(\mathrm{n}=911)\end{array}$ & $\begin{array}{l}\text { Placebo } \\
(n=883)\end{array}$ & $\begin{array}{l}\text { Median, } \\
21 \text { mo }\end{array}$ & \multirow[t]{3}{*}{$\begin{array}{l}\text { Incidence of new-onset AF } \\
\text { in systolic HF was reduced by } \\
\text { eplerenone (vs placebo) from } \\
4.5 \% \text { to } 2.7 \% \text { with a relative } \\
\text { risk reduction of } 42 \% \text {. }\end{array}$} \\
\hline & & $\begin{array}{l}\beta \text {-blocker + ACEI / ARB } \\
\text { therapy }\end{array}$ & & & & \\
\hline & & No history of AF & & & & \\
\hline \multicolumn{7}{|c|}{ Secondary prevention of AF } \\
\hline $\begin{array}{l}\text { Kühlkamp } \\
\text { et al }{ }^{24}\end{array}$ & $\begin{array}{l}\text { Multicenter, } \\
\text { randomized } \\
\text { placebo- } \\
\text {-controlled, } \\
\text { double-blind }\end{array}$ & $\begin{array}{l}n=394 \\
\text { Mean (SD) age, } 60 \text { (12) y } \\
\text { Cardioversion of PeAF } \\
\text { HTN, 46\%-49\%; CHF, } \\
25 \%\end{array}$ & $\begin{array}{l}\text { Metoprolol } \\
\text { CR/XL }(n=197)\end{array}$ & Placebo $(n=197)$ & $\begin{array}{l}\text { Approx. } \\
3 \text { months }\end{array}$ & $\begin{array}{l}\text { Metoprolol compared with } \\
\text { placebo significantly reduced } \\
\text { the risk of recurrence after } \\
\text { electrical or pharmacological } \\
\text { cardioversion of PeAF. }\end{array}$ \\
\hline $\begin{array}{l}\text { Disertori } \\
\text { et al, }{ }^{25} \\
\text { GISSI-AF }\end{array}$ & $\begin{array}{l}\text { Multicenter, } \\
\text { randomized } \\
\text { placebo- } \\
\text {-controlled }\end{array}$ & $\begin{array}{l}n=1442 \\
\text { Mean (SD) age, } 68(9) \\
\text { PAF or cardioverted } \\
\text { PeAF } \\
\text { HTN, } 85 \%\end{array}$ & $\begin{array}{l}\text { Valsartan } \\
(\mathrm{n}=722)\end{array}$ & $\begin{array}{l}\text { Placebo } \\
(n=720)\end{array}$ & $1 \mathrm{y}$ & $\begin{array}{l}\text { Valsartan was not associated } \\
\text { with a reduction in } \\
\text { the incidence of recurrent AF } \\
\text { compared with placebo } \\
\text { (51.4\% vs } 52.1 \%) \text {. }\end{array}$ \\
\hline
\end{tabular}


TABLE 1 Studies evaluating the impact of antihypertensive therapy on the prevention of atrial fibrillation in hypertensive patients (continued from the previous page)

\begin{tabular}{|c|c|c|c|c|c|c|}
\hline Study & Study design & $\begin{array}{l}\text { Study participants } \\
\text { (enrollment criteria) }\end{array}$ & RFM strategy & Control & Follow-up & $\begin{array}{l}\text { Main findings } \\
\text { (AF prevention) }\end{array}$ \\
\hline \multicolumn{7}{|c|}{ Secondary prevention of AF } \\
\hline $\begin{array}{l}\text { Goette } \\
\text { et al, }{ }^{26} \\
\text { ANTIPAF }\end{array}$ & $\begin{array}{l}\text { Multicenter, } \\
\text { randomized } \\
\text { placebo- } \\
\text {-controlled }\end{array}$ & $\begin{array}{l}n=425 \\
\text { Mean (SD) age, } 61 \text { (10) } \\
\text { PAF } \\
\text { Without SHD, HTN 49\% }\end{array}$ & $\begin{array}{l}\text { Olmesartan } \\
(n=214)\end{array}$ & Placebo $(n=211)$ & $1 y$ & $\begin{array}{l}\text { Olmesartan does not reduce } \\
\text { the AF burden and number } \\
\text { of hospitalizations nor } \\
\text { improve quality of life over } \\
\text { placebo. }\end{array}$ \\
\hline $\begin{array}{l}\text { Madrid } \\
\text { et al }{ }^{27}\end{array}$ & $\begin{array}{l}\text { Single-center, } \\
\text { randomized } \\
\text { controlled, } \\
\text { prospective }\end{array}$ & $\begin{array}{l}\mathrm{n}=154 \\
\text { Mean (SD) age, } 66(9) \text { y } \\
\text { PeAF (approx. } 6 \text { mo) } \\
\text { Electrical cardioversion }\end{array}$ & $\begin{array}{l}\text { Irbesartan } \\
+ \text { amiodarone } \\
(\mathrm{n}=79)\end{array}$ & $\begin{array}{l}\text { Amiodarone } \\
(n=75)\end{array}$ & $\begin{array}{l}\text { Median } \\
(\text { IQR), } 254 \\
(60-710) d\end{array}$ & $\begin{array}{l}\text { Addition of irbesartan } \\
\text { to amiodarone increases } \\
\text { the } 6 \text {-month AF-freedom post } \\
\text { cardioversion of PeAF ( } 55.9 \% \\
\text { vs } 79.5 \% \text { ). }\end{array}$ \\
\hline Ueng et $\mathrm{a}^{28}$ & $\begin{array}{l}\text { Single-center, } \\
\text { randomized } \\
\text { controlled, } \\
\text { prospective }\end{array}$ & $\begin{array}{l}\mathrm{n}=145 \\
\text { Mean age across group, } \\
64-66 \text { y } \\
\text { PeAF }>3 \text { mo } \\
\text { Electrical cardioversion }\end{array}$ & $\begin{array}{l}\text { Enalapril } \\
+ \text { amiodarone }\end{array}$ & Amiodarone & $\begin{array}{l}\text { Median } \\
(\text { IQR) } 270 \\
(61-575) d\end{array}$ & $\begin{array}{l}\text { Addition of enalapril } \\
\text { to amiodarone increases } \\
\text { the AF-freedom after } \\
\text { cardioversion of PeAF }(74.3 \% \\
\text { vs } 57.3 \%) \text {. }\end{array}$ \\
\hline $\begin{array}{l}\text { Pokushalov } \\
\text { et al }{ }^{29}\end{array}$ & $\begin{array}{l}\text { Meta-analysis } \\
\text { of } 2 \text { randomized } \\
\text { prospective trials }\end{array}$ & $\begin{array}{l}\mathrm{n}=80 \\
\text { Mean (SD) age, } 56(6) \text { y } \\
\text { Ablation of PAF / PeAF } \\
\text { HTN resistant to } \geq 3 \\
\text { antihypertensive drugs }\end{array}$ & $\begin{array}{l}\text { Renal } \\
\text { denervation } \\
+ \text { PVI }\end{array}$ & PVI alone & $1 y$ & $\begin{array}{l}\text { Renal denervation improves } \\
\text { the results of PVI in patients } \\
\text { with PeAF }(H R, 0.39) \text { and } \\
\text { severe }(\geq 160 / 100 \mathrm{~mm} \mathrm{Hg}) \\
\text { HTN }(0.37) .\end{array}$ \\
\hline $\begin{array}{l}\text { Parkash } \\
\text { et al, }{ }^{31} \\
\text { SMAC-AF }\end{array}$ & $\begin{array}{l}\text { Multicenter, } \\
\text { randomized } \\
\text { prospective, } \\
\text { parallel }\end{array}$ & $\begin{array}{l}\mathrm{n}=143 \\
\text { Mean age } 60 \mathrm{y} \\
\text { Ablation of PAF } / \text { PeAF } \\
B P>130 / 80 \mathrm{~mm} \mathrm{Hg}\end{array}$ & $\begin{array}{l}\text { Aggressive BP } \\
\text { treatment } \\
\text { (target } \\
\text { BP }<120 / 80) \text {, } \\
\text { starting approx. } \\
3 \text { mo prior to } \\
\text { ablation }(n=88)\end{array}$ & $\begin{array}{l}\text { Standard BP } \\
\text { treatment } \\
(<140 / 90 \mathrm{~mm} \mathrm{Hg}) \\
\mathrm{n}=85\end{array}$ & $\begin{array}{l}\text { Median } \\
(\mathrm{IQR}) \\
14(81-27) \\
\text { mo }\end{array}$ & $\begin{array}{l}\text { More aggressive BP treatment } \\
\text { did not reduce the AF } \\
\text { recurrence rate post ablation } \\
\text { but resulted in higher } \\
\text { incidence of hypotension. }\end{array}$ \\
\hline
\end{tabular}

Abbreviations: ACEI, angiotensin-converting enzyme inhibitor; AF, atrial fibrillation; ANTIPAF, Angiotensin II-antagonist in Paroxysmal Atrial Fibrillation; AMI, acute myocardial infarction; ARB, angiotensin II receptor blocker; BP, blood pressure; CCB, calcium channel blocker; CHF, congestive heart failure; DM, diabetes mellitus; ECG, electrocardiogram; EF, ejection fraction; EMPHASIS-HF, Eplerenone in Mild Patients Hospitalization And Survival Study in Heart Failure; GISSI-AF, Gruppo Italiano per lo Studio della Sopravvivenza nell'Infarto Miocardico-Atrial Fibrillation; HF, heart failure; HTN, hypertension; HR, hazard ratio; IQR, interquartile range; LIFE, Losartan Intervention for Endpoint Reduction in Hypertension; LVH, left ventricular hypertrophy; NYHA, New York Heart Association; PAF, paroxysmal atrial fibrillation; PeAF, persistent atrial fibrillation; PVD, peripheral vascular disease; PVI, pulmonary vein isolation; RFM, risk factors modification; SBP, systolic blood pressure; SHD, structural heart disease; SMAC-AF, Substrate Modification with Aggressive Blood Pressure Control

A large Taiwanese cohort of patients with type 2 DM was followed for 13 years. ${ }^{41}$ After adjusting for confounding factors, metformin use was independently associated with a $19 \%$ decrease of the incident AF risk. ${ }^{41}$ Other nationwide cohort study of patients with DM who were not insulin dependent showed a preventive effect of thiazolidinediones (TZDs) on incident AF, which reduced the 5-year AF incidence by $30 \%$ compared with TZD nonusers $(1.2 \%$ vs $1.8 \%$, respectively). ${ }^{42}$ The recent study that included older patients with DM demonstrated a significantly higher incident $\mathrm{AF}$ rate among the insulin users (vs nonusers, OR, 1.58) as well as a lower rate of new-onset $\mathrm{AF}$ among patients using dipeptidyl peptidase- 4 inhibitors (vs nonusers, OR, 0.65). ${ }^{43}$

Although poor glycemia control predicted new-onset $\mathrm{AF}$, the randomized study reported similar 5-year incident AF rates among patients with DM and high cardiovascular risk who underwent the intensive glycol regulation (targeting $\mathrm{HbA}_{1 \mathrm{c}}$ level of $<6 \%$ ) and those who continued with the standard treatment (targeting $\mathrm{HbA}_{1 \mathrm{c}}$ level between $7 \%$ and 7.9\%) (TABLE2). ${ }^{44}$

Secondary prevention It seems that pioglitazidone, one of the TZDs, may protect patients with type $2 \mathrm{DM}$ and paroxysmal AF from recurrent $\mathrm{AF}$ after catheter ablation. ${ }^{45}$ In pioglitazidone users, as compared with nonusers, the 2-year single PV-isolation procedure success was significantly higher $(86.3 \%$ vs $70.7 \%$, respectively), while the need for redo-ablation was significantly lower (9.8\% vs $24.2 \%$, respectively) (TABLE2). ${ }^{45}$

\section{Physical activity Physical activity and the risk of} atrial fibrillation Most trials evaluating the association between physical activity and AF are limited by self-reporting of total exercise level and detection of only documented AF cases. ${ }^{46-51}$ It seems that the impact of physical activity on AF risk is influenced by age, sex, and type of exercise. ${ }^{48,49}$ 
TABLE 2 Studies on the effect of antidiabetic treatment on the prevention of atrial fibrillation in patients with diabetes mellitus

\begin{tabular}{|c|c|c|c|c|c|c|}
\hline Study & Study design & $\begin{array}{l}\text { Study participants } \\
\text { (enrollment criteria) }\end{array}$ & RFM strategy & Control & Follow-up & $\begin{array}{l}\text { Main findings } \\
\text { (AF prevention) }\end{array}$ \\
\hline \multicolumn{7}{|c|}{ Primary prevention of $\mathrm{AF}$} \\
\hline $\begin{array}{l}\text { Chang et al, }{ }^{41} \\
\text { Taiwanese } \\
\text { LHDB } \\
\text { 1999-2010 }\end{array}$ & $\begin{array}{l}\text { Population- } \\
\text {-based, } \\
\text { retrospective, } \\
\text { observational }\end{array}$ & $\begin{array}{l}\mathrm{n}=645710 \\
\text { Mean (SD) age, } \\
59(17) \text { y } \\
\text { Type } 2 \text { DM } \\
\text { No other antidiabetic } \\
\text { therapy except } \\
\text { metformin } \\
\text { No history of AF }\end{array}$ & $\begin{array}{l}\text { Metformin users } \\
(n=85198)\end{array}$ & $\begin{array}{l}\text { Metformin } \\
\text { nonusers } \\
(n=560512)\end{array}$ & $13 y$ & $\begin{array}{l}\text { Lower incidence of new- } \\
\text {-onset AF in metformin } \\
\text { users compared with } \\
\text { nonusers ( } 245 \text { vs } 293 \text { per } \\
100000 \text { person-years); } \\
\text { metformin reduced } \\
\text { the relative risk of incident } \\
\text { AF by } 19 \%\end{array}$ \\
\hline Chao et al ${ }^{42}$ & $\begin{array}{l}\text { Population- } \\
\text {-based, } \\
\text { retrospective, } \\
\text { observational }\end{array}$ & $\begin{array}{l}n=12065 \\
\text { Mean (SD) age, } 54 \\
(12) y \\
\text { Non-insulin dependent } \\
\text { DM }\end{array}$ & TZD users $(n=4137)$ & $\begin{array}{l}\text { TZD nonusers } \\
(\mathrm{n}=7928)\end{array}$ & $\begin{array}{l}\text { Mean (SD), } \\
63(25) \text { mo }\end{array}$ & $\begin{array}{l}\text { Lower rate of incident AF } \\
\text { in TZD users than } \\
\text { in nonusers ( }(1.2 \% \text { vs } 1.8 \%) \text {; } \\
\text { TZDs independently } \\
\text { protected from de novo AF } \\
(\mathrm{HR}, 0.69) \text { in DM }\end{array}$ \\
\hline Chen et al ${ }^{43}$ & $\begin{array}{l}\text { Retrospective, } \\
\text { observational, } \\
\text { nationwide } \\
\text { nested } 1: 4 \\
\text { matched }\end{array}$ & $\begin{array}{l}n=9790 \\
\text { Age } \geq 65 y \\
\text { New-diagnosed DM }\end{array}$ & $\begin{array}{l}\text { Insulin, } 8.2 \% \\
\text { Metformin, } 51.6 \% \\
\text { Acarboses, } 12.2 \% \\
\text { Glinides, } 8.2 \% \\
\text { Sulfonylureas, } 55.9 \% \\
\text { TZDs, } 14.3 \% \\
\text { DPP4 inhibitors, 3.2\% }\end{array}$ & $\begin{array}{l}\text { Nonusers } \\
\text { (as reference) }\end{array}$ & $7 y$ & $\begin{array}{l}\text { In elderly patients with } \\
\text { diabetes, the risk of new- } \\
\text {-onset AF was higher among } \\
\text { the insulin users } \\
\text { (multivariate OR, 1.58) and } \\
\text { was lower among the DPP4 } \\
\text { inhibitor users (OR, 0.65) } \\
\text { compared with nonusers. }\end{array}$ \\
\hline $\begin{array}{l}\text { Fatemi et } \\
\mathrm{al}^{, 44} \mathrm{ACCORD}\end{array}$ & $\begin{array}{l}\text { Multi-center, } \\
\text { randomized, } \\
\text { double-blind, } \\
\text { prospective }\end{array}$ & $\begin{array}{l}\mathrm{n}=10082 \\
\text { High-risk }{ }^{\mathrm{a}} \mathrm{DM} \text { patients } \\
\text { Approx. } 35 \% \text { receiving } \\
\text { insulin } \\
\mathrm{HbA}_{1 \mathrm{c}} \geq 7.5 \%\end{array}$ & $\begin{array}{l}\text { Intensive glycemic } \\
\text { control, targeting } \\
\mathrm{HbA}_{1 \mathrm{C}}<6 \%(\mathrm{n}=5040)\end{array}$ & $\begin{array}{l}\text { Standard } \\
\text { glycemic control, } \\
\mathrm{HbA}_{1 \mathrm{c}} 7 \%-7.9 \% \\
(\mathrm{n}=5042)\end{array}$ & $\begin{array}{l}\text { Median, } \\
4.7 \mathrm{y}\end{array}$ & $\begin{array}{l}\text { Intensive glycemic control } \\
\text { failed to prevent new-onset } \\
\text { AF more than a standard DM } \\
\text { treatment strategy. }\end{array}$ \\
\hline Second & AF & & & & & \\
\hline Gu et al ${ }^{45}$ & $\begin{array}{l}\text { Prospective, } \\
\text { observational }\end{array}$ & $\begin{array}{l}\mathrm{n}=150 \\
\text { Drug-refractory PAF } \\
\text { Catheter ablation (PVI) } \\
\text { Type } 2 \text { DM }\end{array}$ & $\begin{array}{l}\text { TZD (pioglitazone) } \\
\text { users before } \\
\text { ablation }(n=51)\end{array}$ & $\begin{array}{l}\text { TZD (pioglitazone) } \\
\text { nonusers }(n=99)\end{array}$ & $\begin{array}{l}\text { Mean (SD), } \\
23(5) \text { mo }\end{array}$ & $\begin{array}{l}\text { A single ablation success } \\
\text { was better ( } 86.3 \% \text { vs } 70.7 \%) \\
\text { and the rate of redo ablation } \\
\text { was lower }(9.8 \% \text { vs } 24.2 \%) \\
\text { in the pioglitazone users } \\
\text { than nonusers. }\end{array}$ \\
\hline $\begin{array}{l}\text { Donnellan } \\
\text { et al }{ }^{40}\end{array}$ & $\begin{array}{l}\text { Retrospective, } \\
\text { observational }\end{array}$ & $\begin{array}{l}\mathrm{n}=298 \\
\text { Mean (SD) age, } 67(8) \mathrm{y} \\
\text { Type } 1 \mathrm{DM}, 12.1 \% \text { and } \\
\text { type } 2 \mathrm{DM}, 87.9 \% \\
\text { Catheter ablation of AF }\end{array}$ & $\begin{array}{l}\mathrm{HbA}_{4 \mathrm{c}} \text { improvement } \\
\text { during a } 12 \text {-month } \\
\text { period preceding } \\
\mathrm{AF} \text { ablation: } \\
>10 \% \text { reduction, } \\
0 \%-10 \% \text { reduction, or } \\
\text { worsening }\end{array}$ & - & $\begin{array}{l}\text { Mean (SD), } \\
26(20) \text { mo }\end{array}$ & $\begin{array}{l}\text { The proportion decrease } \\
\text { in } \mathrm{HbA}_{1 \mathrm{c}} \text { during a } 12 \text {-month } \\
\text { period preceding catheter- } \\
\text {-ablation was independently } \\
\text { associated with AF-free } \\
\text { survival post ablation } \\
(\mathrm{HR}, 0.714) \text {. }\end{array}$ \\
\hline
\end{tabular}

a High-risk patients: those with cardiovascular disease or aged of 55 to 79 years or those with anatomic evidence of significant atherosclerosis, albuminuria, left ventricular hypertrophy, or 2 additional cardiovascular risk factors (dyslipidemia, hypertension, current smoking status, or obesity)

Abbreviations: ACCORD, Action to Control Cardiovascular Risk in Diabetes; DPP4, dipeptidyl peptidase-4; HbA , $^{\prime}$ hemoglobin A1c; LHDB, Longitudinal Cohort of Diabetes Patients Database; OR, odds ratio; TZD, thiazolidinedione; others, see TABLE1

Thus, a regular leisure-time exercise in younger men increases the lifelong risk of new-onset AF by almost $20 \%,{ }^{48}$ whereas daily walking or cycling in middle-aged men reduces the risk by $12 \% .{ }^{48} \mathrm{How}^{-}$ ever, a prospective study on middle-aged women reported opposite results, suggesting that protective effects of exercise on AF development were lost after adjusting for their body mass index..$^{50}$ The Physicians' Health Study demonstrated that daily jogging (but not cycling, swimming, or racquet sports) increased the long-term risk of incident $\mathrm{AF}$ by $53 \%$ compared with subjects not regularly participating in vigorous exercise. ${ }^{51} \mathrm{In}$ terestingly, in the Copenhagen City Heart Study, a high (ie, walking most of the working hours, often walking upstairs) and very high occupational physical activity (ie, heavy physical work) were associated with a $21 \%$ and $39 \%$ increase in the risk of incident $\mathrm{AF}$, respectively, compared with mostly sedentary work. ${ }^{52}$ 
Baseline exercise tolerance is inversely associated with long-term risk of incident and recurrent AF. ${ }^{53,54}$ Thus, for every $10 \%$ increase in functional aerobic capacity at baseline exercise test, the risk of new-onset AF was reduced by $7 \%$ during the 14 -year follow-up. ${ }^{53}$ This holds true also for patients with previous history of AF. A long-term AF-freedom with or without rhythm-control strategies was significantly better in patients with AF exhibiting high-peak metabolic equivalents (METs; $>100 \%$ of predicted) than in those showing low-peak METs ( $<85 \%$ of predicted) at baseline testing (66\% vs $12 \%$, respectively). ${ }^{55}$

Level of physical activity and the prevention of atrial fibrillation Primary prevention A relationship between the lifetime exposure to exercise and the risk of new-onset AF is complex. ${ }^{56-59}$ Several trials indicate a U-shaped dose-response association between the level of physical activity and AF incidence. ${ }^{56,57}$ In the Cardiovascular Health Study regular exercise reduced the risk of incident $\mathrm{AF}$, but the intensity of physical activity showed a nonlinear relationship with $\mathrm{AF}$ occurrence, wherein the arrhythmia risk was the lowest (HR, 0.72) with moderate level of exercise and significantly higher with both low and high exercise levels (HR, 0.85 and HR, 0.87, respectively). ${ }^{57}$ Other studies found a more linear relationship between exercise level and incident $\mathrm{AF}$, with a decline in long-term risk of new-onset AF across the entire spectrum of various exercise levels, by rate of $4.8 \%$ per each 1 MET hr/d. ${ }^{58}$ On the contrary, the risk of incident $\mathrm{AF}$ increased linearly with intensity of exercise training among competitive athletes. ${ }^{59}$ Available evidence suggests that the exercise level in the range of 1000 to $1500 \mathrm{METs}$ min/wk (ie, roughly 220 to 330 minutes of moderate walking per week) may protect against new-onset AF. ${ }^{60}$ Interestingly, although an improvement in maximal exercise capacity during lifetime was associated with lower risk of all-cause mortality ${ }^{61}$ and heart failure, it did not prevent incident AF (tABLE 3). ${ }^{62}$

Secondary prevention Regular physical activity emerged as an important part of therapy for recurrent $\mathrm{AF} .{ }^{60}$ The potential benefits of yoga on paroxysmal AF treatment was evaluated in the small randomized study. ${ }^{63}$ Practicing yoga for 1 hour twice weekly during 3 months was associated with significant reduction of AF burden assessed by a noninvasive loop-recorder as well as the improvement in quality of life. ${ }^{63}$ Another randomized study reported that patients with recurrent $\mathrm{AF}$ who completed a 3-month exercise program (consisting of 35-minute physician-controlled walking/running sessions performed 3 times per week) significantly reduced the average time in AF detected by implantable loop recorder from $8.1 \%$ to $4.8 \%$, while those who continued their usual physical activities actually increased the time in AF from $10.4 \%$ to $14.6 \% .{ }^{64}$ In addition, the CARDIO-FIT (Impact of Cardiorespiratory Fitness on Arrhythmia Recurrence in Obese Individuals With Atrial Fibrillation) study demonstrated a dose-response relationship between improvement in cardiorespiratory fitness and $\mathrm{AF}$ burden in obese patients participating in a structured exercise program, consisting of at least $200 \mathrm{~min} / \mathrm{wk}$ of low-to-moderate exercise. ${ }^{55}$ Increasing the level of physical exercise to high intensity (ie, $80 \%$ of maximal capacity) adds no clinical benefit over low intensity training (50\% of maximal exertion) with respect to AF burden and hospitalizations (TABLE3). ${ }^{65}$

\section{Cigarette smoking Cigarette smoking and the} risk of atrial fibrillation Approximately $25 \%$ of adult men and $20 \%$ of women are currently declared as cigarette smokers. ${ }^{66}$ Although epidemiological studies provided inconsistent findings, in several large population-based cohorts, smoking status at baseline was associated with an increased risk of incident AF during follow-up by almost $40 \% .{ }^{67-69}$ Moreover, $6.7 \%$ and $1.4 \%$ of total AF risk in men and women, respectively, can be attributed to tobacco use. ${ }^{67}$ Even an early exposure to second-hand smoke during childhood increase the risk of AF later in life by $40 \%{ }^{70}$

Duration and intensity of smoking affect the risk of new-onset AF. Thus, AF incidence was directly related to years of previous smoking exposure. ${ }^{69}$ The incident AF risk was significantly higher in heavy smokers (>15 g/d of tobacco) than among light-to-moderate smokers (1-14 g/d). ${ }^{71}$ A recent study suggested a more complex dose-response relationship because plasma level of nicotine metabolite, cotinine, which is strongly associated with AF occurrence, rises steeply with consumption of first 10 cigarettes per day, but then reaches a plateau. ${ }^{72}$

Among patients with recurrent AF, smokers have a higher risk of arrhythmia relapse after cardioversion and catheter ablation compared with never smokers. In a large prospective study, the risk of AF recurrence at 1 year following cardioversion was independently associated with the baseline smoking status in elderly women (vs nonsmokers, HR, 1.71), but not in men. ${ }^{73}$ Furthermore, the 1-year AF recurrence rate after $\mathrm{PV}$-isolation was significantly higher in smokers than in nonsmokers (43\% vs $14 \%$; HR, 3.19). ${ }^{74}$

Smoking cessation and the prevention of atrial fibrillation Primary prevention Data regarding the effects of smoking cessation on the prevention of incident AF are conflicting. The Rotterdam Study suggests that persons who quit smoking remain at the increased risk of new-onset $\mathrm{AF}$ similar to 
TABLE 3 Studies on dose-response between physical activity and the risk of atrial fibrillation

\begin{tabular}{|c|c|c|c|c|c|c|}
\hline Study & Study design & $\begin{array}{l}\text { Study participants } \\
\text { (enrollment } \\
\text { criteria) }\end{array}$ & RFM strategy & Control & Follow-up & $\begin{array}{l}\text { Main findings } \\
\text { (AF prevention) }\end{array}$ \\
\hline \multicolumn{7}{|c|}{ Primary prevention of AF } \\
\hline $\begin{array}{l}\text { Morseth et al, }{ }^{56} \\
\text { Tromsø } 3\end{array}$ & $\begin{array}{l}\text { Community-based, } \\
\text { prospective, } \\
\text { longitudinal, } \\
\text { observational }\end{array}$ & $\begin{array}{l}\mathrm{n}=20484 \\
\text { Mean age across } \\
\text { group, } 36-39 \text { y } \\
\text { No previous AF } \\
\text { Leisure time PA } \\
\text { data 7y prior to } \\
\text { inclusion }\end{array}$ & $\begin{array}{l}\text { Low PA: sedentary } \\
\text { lifestyle } \\
\text { Moderate PA: } \geq 4 \mathrm{~h} / \text { wk } \\
\text { of PA (cycling, walking) } \\
\text { High PA: } \geq 4 \mathrm{~h} / \text { wk of } \\
\text { recreational sports } \\
\text { Vigorous PA: } \\
\text { competitive sports }\end{array}$ & - & Mean, $20 \mathrm{y}$ & $\begin{array}{l}\text { U-shaped dose- } \\
\text {-response between PA } \\
\text { level and new AF risk } \\
\text { Those with moderate } \\
\text { PA had a } 19 \% \text { lower risk } \\
\text { of incident AF } \\
\text { compared with those } \\
\text { with low and high PA. }\end{array}$ \\
\hline Williams et a ${ }^{58}$ & $\begin{array}{l}\text { Population-based, } \\
\text { prospective, } \\
\text { longitudinal, } \\
\text { observational }\end{array}$ & $\begin{array}{l}\mathrm{n}=46807 \\
\text { Mean age across } \\
\text { group, } 44-59 \mathrm{y} \\
\text { Recreational } \\
\text { runners and } \\
\text { walkers } \\
\text { No previous AF }\end{array}$ & $\begin{array}{l}\text { Baseline questionnaire } \\
\text { on running and walking } \\
\text { history } \\
\text { Light PA: <3 METs } \\
\text { Moderate PA: } 3-6 \text { METs } \\
\text { Vigorous PA: > } 6 \text { METs }\end{array}$ & - & Mean, $6.2 \mathrm{y}$ & $\begin{array}{l}\text { A linear decline in the } \\
\text { relative risk of incident } \\
\text { cardiac arrhythmias by } \\
\text { reported level of PA: } \\
\text { each } 1 \mathrm{MET} \text { hr/d } \\
\text { increment was } \\
\text { associated with } \\
\text { the } 4.8 \% \text { risk reduction. }\end{array}$ \\
\hline Andersen et al ${ }^{59}$ & $\begin{array}{l}\text { Population-based, } \\
\text { prospective, } \\
\text { longitudinal, } \\
\text { observational }\end{array}$ & $\begin{array}{l}\mathrm{n}=52755 \\
\text { Mean (SD) age, } 39 \\
(12) \mathrm{y} \\
\text { Former } \\
\text { participants in } \\
\text { the } 90 \mathrm{~km} \text { cross- } \\
\text {-country skiing } \\
\text { race } \\
\text { No AF at baseline }\end{array}$ & $\begin{array}{l}\text { Evaluation of } \\
\text { the association } \\
\text { between the number of } \\
\text { completed races during } \\
\text { sports career and } \\
\text { the risk of subsequent } \\
\text { cardiac arrhythmias }\end{array}$ & - & Median, 9.7y & $\begin{array}{l}\text { Former athletes } \\
\text { showed a linear rise in } \\
\text { the future risk of new } \\
\text { AF by the number of } \\
\text { finished races: the risk } \\
\text { was } 22 \%, 27 \% \text {, and } \\
29 \% \text { in participants } \\
\text { who completed } 2,3-4 \text {, } \\
\text { and } \geq 5 \text { races, } \\
\text { respectively. }\end{array}$ \\
\hline Secondary prevent & on of $\mathrm{AF}$ & & & & & \\
\hline Lakkireddy et al ${ }^{63}$ & $\begin{array}{l}\text { Single-center, } \\
\text { prospective, pre- } \\
\text {-post cohort study }\end{array}$ & $\begin{array}{l}\mathrm{n}=52 \\
\text { Mean (SD) age, } 61 \\
(11) \mathrm{y} \\
\text { Symptomatic PAF } \\
\text { No AADs change } \\
\text { during the study }\end{array}$ & $\begin{array}{l}\text { Control phase ( } 3 \text { mo) } \\
\text { followed by yoga phase } \\
\text { (next } 3 \text { mo) } \\
\text { Twice-weekly } 60 \text {-min } \\
\text { yoga training } \\
\text { AF detection by cardiac } \\
\text { external event } \\
\text { monitors }\end{array}$ & None & $3 \mathrm{mo}$ & $\begin{array}{l}\text { Yoga reduced } \\
\text { symptomatic AF } \\
\text { attacks (mean [SD], } \\
3.8[3] \text { vs } 2.1 \text { [2.6]) and } \\
\text { improved the QoL. In } \\
22 \% \text { of patients, no AF } \\
\text { episode was recorded } \\
\text { during the yoga } \\
\text { intervention phase. }\end{array}$ \\
\hline Malmo et al ${ }^{64}$ & $\begin{array}{l}\text { Single-center, } \\
\text { randomized, } \\
\text { prospective }\end{array}$ & $\begin{array}{l}\mathrm{n}=51 \\
\text { Mean age across } \\
\text { group, 56-62 y } \\
\text { Symptomatic } \\
\text { PAF/PeAF } \\
\text { (referred for CA of } \\
\text { AF) } \\
\text { Implanted loop } \\
\text { recorder }\end{array}$ & $\begin{array}{l}\text { The 12-week aerobic } \\
\text { interval training, } \\
\text { consisting of four } \\
4 \text {-min intervals } \\
\text { at } 85 \%-95 \% \text { of peak } \\
\text { heart rate } 3 \text {-times per } \\
\text { week }(n=26)\end{array}$ & $\begin{array}{l}\text { Controls, } \\
\text { patients } \\
\text { continuing } \\
\text { usual } \\
\text { exercise } \\
\text { habits } \\
(n=25)\end{array}$ & $4 \mathrm{mo}$ & $\begin{array}{l}\text { 12-week structured } \\
\text { aerobic interval } \\
\text { training reduced } \\
\text { the mean time in AF } \\
\text { (from } 8.1 \% \text { to } 4.8 \% \text { ) } \\
\text { and improved } \\
\text { symptoms of AF and } \\
\text { QoL. }\end{array}$ \\
\hline $\begin{array}{l}\text { Pathak, et al, }, 55 \\
\text { CARDIO-FIT }\end{array}$ & $\begin{array}{l}\text { Single-center, } \\
\text { observational, } \\
\text { prospective, } \\
\text { longitudinal }\end{array}$ & $\begin{array}{l}\mathrm{n}=308 \\
\text { Mean age across } \\
\text { group, } 58-69 \mathrm{y} \\
\mathrm{BMI} \geq 27 \mathrm{~kg} / \mathrm{m}^{2} \\
\text { PAF and PeAF }\end{array}$ & $\begin{array}{l}\text { A goal-directed } \\
\text { program: } \\
\text { Tailored diet and CR } \\
\text { fitness aiming to } \\
\text { reduce weight by } \geq 10 \% \\
\text { and BMI to } \leq 25 \mathrm{~kg} / \mathrm{m}^{2} \\
\text { Risk factors therapy }\end{array}$ & None & $\begin{array}{l}\text { Mean (SD), } \\
49(19) \text { mo }\end{array}$ & $\begin{array}{l}\text { AF-freedom and } \\
\text { symptomatic AF } \\
\text { burden were better } \\
\text { with CR fitness gain } \geq 2 \\
\text { METs (Vs <2 METs) } \\
\text { CR fitness enhances } \\
\text { the benefits of weight } \\
\text { loss on AF outcome } \\
\text { (1 MET gain was } \\
\text { associated with a 9\% } \\
\text { decline in AF } \\
\text { recurrence rate) }\end{array}$ \\
\hline
\end{tabular}

Abbreviations: AAD, antiarrhythmic drugs; BMI, body mass index; CA, catheter ablation; CARDIO-FIT, Impact of Cardiorespiratory Fitness on Arrhythmia Recurrence in Obese Individuals With Atrial Fibrillation; CR, cardiorespiratory; MET, metabolic equivalent; PA, physical activity; QoL, quality of life; others, see TABLES 1 and 2 
TABLE 4 Studies on the relationship between smoking modification and the risk of atrial fibrillation

\begin{tabular}{|c|c|c|c|c|c|c|}
\hline Study & Study design & $\begin{array}{l}\text { Study participants } \\
\text { (enrollment } \\
\text { criteria) }\end{array}$ & RFM strategy & Control & Follow-up & $\begin{array}{l}\text { Main findings } \\
\text { (AF prevention) }\end{array}$ \\
\hline \multicolumn{7}{|c|}{ Primary prevention of AF } \\
\hline Zhu et al ${ }^{67}$ & $\begin{array}{l}\text { Meta-analysis of } 16 \\
\text { prospective studies }\end{array}$ & $\begin{array}{l}\mathrm{n}=286217 \\
\text { Age range, 39-94 y } \\
\text { No prevalent AF } \\
\text { Available data on } \\
\text { smoking history }\end{array}$ & $\begin{array}{l}\text { Smoking } \\
\text { cessation } \\
\text { (current smokers } \\
\text { vs former } \\
\text { smokers }\end{array}$ & $\begin{array}{l}\text { Never } \\
\text { smokers }\end{array}$ & Range, 2-50y & $\begin{array}{l}\text { The incident AF risk } \\
\text { in current and former } \\
\text { smokers was } 39 \% \text { and } \\
16 \% \text {, respectively, } \\
\text { compared with never } \\
\text { smokers. } \\
\text { The AF risk due } \\
\text { to smoking was higher } \\
\text { in men than in women. }\end{array}$ \\
\hline $\begin{array}{l}\text { Wilhelmsen } \\
\text { et al } l^{71}\end{array}$ & $\begin{array}{l}\text { General male } \\
\text { population } \\
\text { (random sample), } \\
\text { prospective }\end{array}$ & $\begin{array}{l}\mathrm{n}=7495 \\
\text { Age range, } 47-55 \mathrm{y} \\
\text { Available smoking } \\
\text { history data }\end{array}$ & $\begin{array}{l}\text { Current smokers } \\
\text { (daily tobacco } \\
\text { intake: } 1-14 \mathrm{~g} \\
\text { vs }>15 \mathrm{~g} \text { ) }\end{array}$ & $\begin{array}{l}\text { Never + ex- } \\
\text {-smokers }\end{array}$ & Mean, 25.2 y & $\begin{array}{l}\text { Risk of hospitalization for } \\
\text { new AF was higher among } \\
\text { heavy smokers (tobacco } \\
\text { intake of }>15 \mathrm{~g} / \mathrm{d} \text { ) than } \\
\text { among light-to-moderate } \\
\text { smokers }(1-14 \mathrm{~g} / \mathrm{d}) \text {. }\end{array}$ \\
\hline $\begin{array}{l}\text { Zuo et al, } 72 \\
\text { Hordaland } \\
\text { Health Study }\end{array}$ & $\begin{array}{l}\text { Population-based, } \\
\text { prospective, } \\
\text { observational }\end{array}$ & $\begin{array}{l}n=6682 \\
\text { Age range, } 46-74 \text { y } \\
\text { Without known AF } \\
\text { Measurement of } \\
\text { plasma cotinine level } \\
\text { at baseline }\end{array}$ & $\begin{array}{l}\text { Current smokers } \\
\text { (categorized by } \\
\text { plasma cotinine } \\
\text { concentration) }\end{array}$ & $\begin{array}{l}\text { Never } \\
\text { smokers and } \\
\text { former } \\
\text { smokers }\end{array}$ & Median, 11 y & $\begin{array}{l}\text { The relationship between } \\
\text { smoking intensity and } \\
\text { plasma cotinine was } \\
\text { nonlinear, reaching } \\
\text { a plateau } \\
\text { at } 15 \text { cigarettes } / \mathrm{d} \text {. } \\
\text { A } 40 \% \text { increase in risk } \\
\text { of new AF in participants } \\
\text { with plasma cotinine } \\
\text { level } \geq 85 \mathrm{vs}<85 \mathrm{nmol} / \mathrm{l} \text {. }\end{array}$ \\
\hline \multicolumn{7}{|c|}{ Secondary prevention of AF } \\
\hline Kinoshita et al ${ }^{79}$ & $\begin{array}{l}\text { Single-center, } \\
\text { prospective, } \\
\text { observational }\end{array}$ & $\begin{array}{l}n=1424 \\
\text { Mean (SD) age, } \\
70(12) \text { y } \\
\text { Consecutive patients } \\
\text { undergoing index } \\
\text { cardioversion for } \\
\text { AF/AFL }\end{array}$ & $\begin{array}{l}\text { Ex-smokers } \\
(n=536), \text { current } \\
\text { smokers }(n=113)\end{array}$ & $\begin{array}{l}\text { Nonsmokers } \\
(n=664)\end{array}$ & $1 y$ & $\begin{array}{l}\text { The 1-year arrhythmia } \\
\text { recurrence rate post } \\
\text { cardioversion among } \\
\text { women was significantly } \\
\text { higher in current smokers } \\
\text { compared with ex- } \\
\text {-smokers ( } 76 \% \text { vs } 61 \%) \text {. }\end{array}$ \\
\hline Fukamizu et al ${ }^{74}$ & $\begin{array}{l}\text { Single-center, } \\
\text { prospective, } \\
\text { observational }\end{array}$ & $\begin{array}{l}n=59 \\
\text { Mean (SD) age, } \\
60(11) \text { y } \\
\text { Consecutive patients } \\
\text { undergoing index } \\
\text { PVI ablation } \\
\text { procedure for drug- } \\
\text {-resistant AF }\end{array}$ & $\begin{array}{l}\text { Former smokers } \\
(n=15), \text { current } \\
\text { smokers }(n=15)\end{array}$ & $\begin{array}{l}\text { Never- } \\
\text {-smokers } \\
(n=30)\end{array}$ & $\begin{array}{l}\text { Mean (SD), } \\
306(95) d\end{array}$ & $\begin{array}{l}\text { The AF recurrence rate } \\
\text { after PVI ablation was } \\
\text { significantly higher } \\
\text { among former smokers } \\
\text { than among never- } \\
\text {-smokers ( } 47 \% \text { vs } 14 \%) \text {. }\end{array}$ \\
\hline
\end{tabular}

Abbreviations: AFL, atrial flutter; others, see TABLES 1-3

current smokers (relative risk, 1.49 and 1.51, respectively, with never smokers used as the reference) ${ }^{69}$ However, the ARIC (Atherosclerosis Risk in Communities) study presented more encouraging conclusions, reporting a significantly lower HR for incident AF in former compared with current smokers (1.32 vs 2.05 , respectively). ${ }^{68}$ Nevertheless, former smokers remained at increased risk for AF development as compared with never smokers. ${ }^{67}$ Therefore, with respect to the primary prevention of AF, it is important never to start smoking (TABLE 4).

Secondary prevention It seems that smoking cessation does not seemingly improve outcome of rhythm control strategies in patients with recurrent AF. The 1-year AF recurrence rate following cardioversion of persistent $\mathrm{AF}$ and catheter ablation of recurrent $\mathrm{AF}$ was similar among current (58\% vs $61 \%$, respectively) and former smokers ( $47 \%$ and $40 \%$, respectively) (TABLE 4). ${ }^{73,74}$

Conclusions Long-term history of hypertension, DM, vigorous or low physical activity, and cigarette smoking are associated with an increased lifetime risk of new-onset AF as well as the risk of relapse of AF following cardioversion or catheter ablation. Optimal management of hypertension with ACEIs, ARBs, and $\beta$-blockers may prevent new-onset $\mathrm{AF}$ and recurrence of $\mathrm{AF}$ 
after cardioversion and ablation. The use of metformin or TZDs could be helpful in the primary prevention of $\mathrm{AF}$ among patients with $\mathrm{DM}$, but more intense glycemia control provides no advantage in the prevention of incident AF compared with standard therapy of diabetes. However, well-managed long-term glycemic control before the intervention may reduce recurrent AF after catheter ablation. Physical activity, consisting of regular moderate exercise may protect against new-onset and recurrent AF. History of former or active cigarette smoking significantly reduces the efficacy of the rhythm control strategies.

\section{ARTICLE INFORMATION}

\section{CONFLICT OF INTEREST None declared}

OPEN ACCESS This is an Open Access article distributed under the terms of the Creative Commons Attribution-NonCommercial-NoDerivatives 4.0 International License (CC BY-NC-ND 4.0), allowing third parties to download articles and share them with others, provided the original work is properly cited, not changed in any way, distributed under the same license, and used for noncommercial purposes only. For commercial use, please contact the journal office at kardiologiapolska@ptkardio.pl.

HOW TO CITE Mujović N, Marinković M, Mihajlović M, et al. Risk factor modification for the primary and secondary prevention of atrial fibrillation. Part 1. Kardiol Pol. 2020; 78: 181-191. doi:10.33963/KP.15221

\section{REFERENCES}

1 Kirchhof P, Benussi S, Kotecha D, et al. 2016 ESC guidelines for the management of atrial fibrillation developed in collaboration with EACTS. Eur Heart J. 2016; 37: 2893-2962.

2 Calkins H, Hindricks G, Cappato R, et al. 2017 HRS/EHRA/ECAS/APHRS/SOLAECE expert consensus statement on catheter and surgical ablation of atrial fibrillation. Heart Rhythm. 2017; 14: e275-e444.

3 Potpara TS, Mujovic N, Lip GYH. Meeting the unmet needs to improve management and outcomes of patients with atrial fibrillation: fitting global solutions to local settings. Pol Arch Intern Med. 2019; 129: 574-576.

4 Gorenek B, Pelliccia A, Benjamin EJ, et al. European Heart Rhythm Association (EHRA)/European Association of Cardiovascular Prevention and Rehabilitation (EACPR) position paper on how to prevent atrial fibrillation endorsed by the Heart Rhythm Society (HRS) and Asia Pacific Heart Rhythm Society (APHRS). Europace. 2017; 19: 190-225.

5 Du X, Dong J, Ma C. Is atrial fibrillation a preventable disease? J Am Coll Cardiol. 2017; 69: 1968-1982.

6 Panchal G, Mahmood M, Lip GYH. Revisiting the risks of incident atrial fibrillation: a narrative review. Part 1. Kardiol Pol. 2019; 77: 430-436.

7 Manolis AJ, Rosei EA, Coca A, et al. Hypertension and atrial fibrillation: diagnostic approach, prevention and treatment. Position paper of the Working Group 'Hypertension Arrhythmias and Thrombosis' of the European Society of Hypertension. J Hypertens. 2012; 30: 239-252.

8 Ogunsua AA, Shaikh AY, Ahmed M, et al. Atrial fibrillation and hypertension: mechanistic, epidemiologic, and treatment parallels. Methodist Debakey Cardiovasc J. 2015; 11: 228-234.

9 Hering D, Szymański FM. Comparison of hypertension epidemiology and treatment in Poland and Australia. Kardiol Pol. 2018; 76: 520-528.

10 Lau YF, Yiu KH, Siu CW, et al. Hypertension and atrial fibrillation: epidemiology, pathophysiology and therapeutic implications. J Hum Hypertens. 2012; 26: $563-569$.

11 Grundvold I, Skretteberg PT, Liestol K, et al. Upper normal blood pressures predict incident atrial fibrillation in healthy middle-aged men: a 35-year follow-up study. Hypertension. 2012; 59: 198-204.

12 Conen D, Tedrow UB, Koplan BA, et al. Influence of systolic and diastolic blood pressure on the risk of incident atrial fibrillation in women. Circulation. 2009; 119: 2146-2152.

13 Thomas MC, Dublin S, Kaplan RC, et al. Blood pressure control and risk of incident atrial fibrillation. Am J Hypertens. 2008; 21: 1111-1116.

14 Fumagalli S, Giannini I, Pupo S, et al. Atrial fibrillation after electrical cardioversion in elderly patients: a role for arterial stiffness? Results from a preliminary study. Aging Clin Exp Res. 2016; 28: 1273-1277.

15 Bhargava M, Di Biase L, Mohanty P, et al. Impact of type of atrial fibrillation and repeat catheter ablation on long-term freedom from atrial fibrillation: results from a multicenter study. Heart Rhythm. 2009; 6: 1403-1412.
16 Letsas KP, Weber R, Burkle G, et al. Pre-ablative predictors of atrial fibrillation recurrence following pulmonary vein isolation: the potential role of inflammation. Europace. 2009; 11: 158-163.

17 Okin PM, Hille DA, Larstorp AC, et al. Effect of lower on-treatment systolic blood pressure on the risk of atrial fibrillation in hypertensive patients. Hypertension. 2015; 66: 368-373.

18 Cohen DL, Townsend RR. Blood pressure in patients with atrial fibrillation: part 2 - treatment. J Clin Hypertens (Greenwich). 2017; 19: 212-214.

19 Marott SC, Nielsen SF, Benn M, et al. Antihypertensive treatment and risk of atrial fibrillation: a nationwide study. Eur Heart J. 2014; 35: 1205-1214.

20 Schaer BA, Schneider C, Jick SS, et al. Risk for incident atrial fibrillation in patients who receive antihypertensive drugs: a nested case-control study. Ann Intern Med. 2010; 152: 78-84.

21 Zhang Y, Zhang P, Mu Y, et al. The role of renin-angiotensin system blockade therapy in the prevention of atrial fibrillation: a meta-analysis of randomized controlled trials. Clin Pharmacol Ther. 2010; 88: 521-531.

22 Nasr IA, Bouzamondo A, Hulot JS, et al. Prevention of atrial fibrillation onset by beta-blocker treatment in heart failure: a meta-analysis. Eur Heart J. 2007; 28: 457-462.

23 Swedberg K, Zannad F, McMurray J], et al. Eplerenone and atrial fibrillation in mild systolic heart failure: results from the EMPHASIS-HF (Eplerenone in Mild Patients Hospitalization And SurvIval Study in Heart Failure) study. J Am Coll Cardiol. 2012; 59: 1598-1603.

24 Kuhlkamp V, Schirdewan A, Stangl K, et al. Use of metoprolol CR/XL to maintain sinus rhythm after conversion from persistent atrial fibrillation: a randomized, double-blind, placebo-controlled study. J Am Coll Cardiol. 2000; 36: 139-146.

25 Disertori M, Latini R, Barlera S, et al. Valsartan for prevention of recurrent atrial fibrillation. N Engl J Med. 2009; 360: 1606-1617.

26 Goette A, Schon N, Kirchhof P, et al. Angiotensin II-antagonist in paroxysmal atrial fibrillation (ANTIPAF) trial. Circ Arrhythm Electrophysiol. 2012; 5: 43-51.

27 Madrid AH, Bueno MG, Rebollo JM, et al. Use of irbesartan to maintain sinus rhythm in patients with long-lasting persistent atrial fibrillation: a prospective and randomized study. Circulation. 2002; 106: 331-336.

28 Ueng KC, Tsai TP, Yu WC, et al. Use of enalapril to facilitate sinus rhythm maintenance after external cardioversion of long-standing persistent atrial fibrillation. Results of a prospective and controlled study. Eur Heart J. 2003; 24: 2090-2098.

29 Pokushalov E, Romanov A, Katritsis DG, et al. Renal denervation for improving outcomes of catheter ablation in patients with atrial fibrillation and hypertension: early experience. Heart Rhythm. 2014; 11: 1131-1138.

30 Rienstra M, Hobbelt AH, Alings M, et al. Targeted therapy of underlying conditions improves sinus rhythm maintenance in patients with persistent atrial fibrillation: results of the RACE 3 trial. Eur Heart J. 2018; 39: 2987-2996.

31 Parkash R, Wells GA, Sapp JL, et al. Effect of aggressive blood pressure control on the recurrence of atrial fibrillation after catheter ablation: a randomized open-label clinical trial (SMAC-AF [Substrate Modification With Aggressive Blood Pressure Control]). Circulation. 2017; 135: 1788-1798.

32 Huxley RR, Filion KB, Konety $\mathrm{S}$, et al. Meta-analysis of cohort and case-control studies of type 2 diabetes mellitus and risk of atrial fibrillation. Am J Cardiol. 2011; 108: 56-62.

33 Nichols GA, Reinier K, Chugh SS. Independent contribution of diabetes to increased prevalence and incidence of atrial fibrillation. Diabetes Care. 2009; 32: 1851-1856

34 Pallisgaard JL, Schjerning AM, Lindhardt TB, et al. Risk of atrial fibrillation in diabetes mellitus: A nationwide cohort study. EurJ Prev Cardiol. 2016; 23: 621-627. 35 Dublin S, Glazer NL, Smith NL, et al. Diabetes mellitus, glycemic control, and risk of atrial fibrillation. J Gen Intern Med. 2010; 25: 853-858.

36 Latini R, Staszewsky L, Sun JL, et al. Incidence of atrial fibrillation in a population with impaired glucose tolerance: the contribution of glucose metabolism and other risk factors. A post hoc analysis of the Nateglinide and Valsartan in Impaired Glucose Tolerance Outcomes Research trial. Am Heart J. 2013; 166: 935-940.e1.

37 Potpara T, Marinkovic-Eric J, Grujic M, et al. Effect of diabetes mellitus in recovery and maintenance of sinus rhythm in patients with persistent atrial fibrillation [in Serbian]. Srp Arh Celok Lek. 2002; 130: 189-192.

38 Soran $\mathrm{H}$, Younis N, Currie P, et al. Influence of diabetes on the maintenance of sinus rhythm after a successful direct current cardioversion in patients with atrial fibrillation. QJM. 2008; 101: 181-187.

39 Anselmino M, Matta M, D'Ascenzo F, et al. Catheter ablation of atrial fibrillation in patients with diabetes mellitus: a systematic review and meta-analysis. Europace. 2015; 17: 1518-1525.

40 Donnellan E, Aagaard P, Kanj M, et al. Association between pre-ablation glycemic control and outcomes among patients with diabetes undergoing atrial fibrillation ablation. JACC Clin Electrophysiol. 2019; 5: 897-903.

41 Chang SH, Wu LS, Chiou MJ, et al. Association of metformin with lower atrial fibrillation risk among patients with type 2 diabetes mellitus: a population-based dynamic cohort and in vitro studies. Cardiovasc Diabetol. 2014; 13: 123.

42 Chao TF, Leu HB, Huang $\mathrm{CC}$, et al. Thiazolidinediones can prevent new onset atrial fibrillation in patients with non-insulin dependent diabetes. Int J Cardiol. 2012; 156: 199-202. 
43 Chen HY, Yang FY, Jong GP. Antihyperglycemic drugs use and new-onset atrial fibrillation in elderly patients. 2017; 47: 388-393.

44 Fatemi 0 , Yuriditsky E, Tsioufis C, et al. Impact of intensive glycemic control on the incidence of atrial fibrillation and associated cardiovascular outcomes in patients with type 2 diabetes mellitus (from the Action to Control Cardiovascular Risk in Diabetes Study). Am J Cardiol. 2014; 114: 1217-1222.

45 Gu J, Liu X, Wang X, et al. Beneficial effect of pioglitazone on the outcome of catheter ablation in patients with paroxysmal atrial fibrillation and type 2 diabetes mellitus. Europace. 2011; 13: 1256-1261.

46 Evenson KR, Butler EN, Rosamond WD. Prevalence of physical activity and sedentary behavior among adults with cardiovascular disease in the United States. J Cardiopulm Rehabil Prev. 2014; 34: 406-419.

47 An R, Xiang $X$, Yang $Y$, et al. Mapping the prevalence of physical inactivity in U.S. states, 1984-2015. PLoS One. 2016; 11:e0 168175.

48 Drca N, Wolk A, Jensen-Urstad M, et al. Atrial fibrillation is associated with different levels of physical activity levels at different ages in men. Heart. 2014; 100: 1037-1042.

49 Drca N, Wolk A, Jensen-Urstad M, et al. Physical activity is associated with a reduced risk of atrial fibrillation in middle-aged and elderly women. Heart. 2015; 101: 1627-1630.

50 Everett BM, Conen D, Buring JE, et al. Physical activity and the risk of incident atrial fibrillation in women. Circ Cardiovasc Qual Outcomes. 2011; 4: 321-327.

51 Aizer A, Gaziano JM, Cook NR, et al. Relation of vigorous exercise to risk of atrial fibrillation. Am J Cardiol. 2009; 103: 1572-1577.

52 Skielboe AK, Marott JL, Dixen U, et al. Occupational physical activity, but no leisure-time physical activity increases the risk of atrial fibrillation: the Copenhagen City Heart Study. EurJ Prev Cardiol. 2016; 23: 1883-1893.

53 Hussain N, Gersh B], Gonzalez Carta K, et al. Impact of cardiorespiratory fitness on frequency of atrial fibrillation, stroke, and all-cause mortality. Am J Cardiol. 2018; 121: 41-49.

54 Qureshi WT, Alirhayim Z, Blaha M], et al. Cardiorespiratory fitness and risk of incident atrial fibrillation: results from the Henry Ford Exercise Testing (FIT) project. Circulation. 2015; 131: 1827-1834.

55 Pathak RK, Elliott A, Middeldorp ME, et al. Impact of CARDIOrespiratory FITness on Arrhythmia Recurrence in Obese Individuals With Atrial Fibrillation: the CARDI0-FIT study. J Am Coll Cardiol. 2015; 66: 985-996.

56 Morseth B, Graff-Iversen S, Jacobsen BK, et al. Physical activity, resting heart rate, and atrial fibrillation: the Tromsø Study. Eur Heart J. 2016; 37: 2307-2313.

57 Mozaffarian D, Furberg CD, Psaty BM, et al. Physical activity and incidence of atrial fibrillation in older adults: the cardiovascular health study. Circulation. 2008; 118: 800-807.

58 Williams PT, Franklin BA. Reduced incidence of cardiac arrhythmias in walkers and runners. PLoS One. 2013; 8: e65302.

59 Andersen K, Farahmand B, Ahlbom A, et al. Risk of arrhythmias in 52755 long-distance cross-country skiers: a cohort study. Eur Heart J. 2013; 34: 3624-3631.

60 Elliott AD, Maatman B, Emery MS, et al. The role of exercise in atrial fibrillation prevention and promotion: finding optimal ranges for health. Heart Rhythm. 2017; 14: 1713-1720.

61 Brawner CA, Al-Mallah MH, Ehrman JK, et al. Change in maximal exercise capacity is associated with survival in men and women. Mayo Clin Proc. 2017; 92: 383-390.

62 Khan H, Kunutsor SK, Rauramaa R, et al. Long-term change in cardiorespiratory fitness in relation to atrial fibrillation and heart failure (from the Kuopio Ischemic Heart Disease Risk Factor Study). Am J Cardiol. 2018; 121: 956-960.

63 Lakkireddy D, Atkins D, Pillarisetti J, et al. Effect of yoga on arrhythmia burden, anxiety, depression, and quality of life in paroxysmal atrial fibrillation: the YOGA My Heart Study. J Am Coll Cardiol. 2013; 61: 1177-1182.

64 Malmo V, Nes BM, Amundsen BH, et al. Aerobic interval training reduces the burden of atrial fibrillation in the short term: a randomized trial. Circulation. 2016; 133: 466-473

65 Skielboe AK, Bandholm TQ, Hakmann S, et al. Cardiovascular exercise and burden of arrhythmia in patients with atrial fibrillation - a randomized controlled trial. PLoS One. 2017; 12: e0170060.

66 National Center for Chronic Disease Prevention and Health Promotion (US) Office on Smoking and Health. Preventing tobacco use among youth and young adults: a report of the Surgeon General. Atlanta, GA: Centers for Disease Control and Prevention (US); 2012.

67 Zhu W, Yuan P, Shen Y, et al. Association of smoking with the risk of incident atrial fibrillation: a meta-analysis of prospective studies. Int J Cardiol. 2016; 218: 259-266

68 Chamberlain AM, Agarwal SK, Folsom AR, et al. Smoking and incidence of atrial fibrillation: results from the Atherosclerosis Risk in Communities (ARIC) study. Heart Rhythm. 2011; 8: 1160-1166

69 Heeringa J, Kors JA, Hofman A, et al. Cigarette smoking and risk of atrial fibrillation: the Rotterdam Study. Am Heart J. 2008; 156: 1163-1169.

70 Dixit S, Pletcher M], Vittinghoff E, et al. Secondhand smoke and atrial fibrillation: data from the Health eHeart Study. Heart Rhythm. 2016; 13: 3-9.
71 Wilhelmsen L, Rosengren A, Lappas G. Hospitalizations for atrial fibrillation in the general male population: morbidity and risk factors. J Intern Med. 2001; 250: 382-389.

72 Zuo H, Nygard O, Vollset SE, et al. Smoking, plasma cotinine and risk of atrial fibrillation: the Hordaland Health Study. J Intern Med. 2018; 283: 73-82.

73 Kinoshita M, Herges RM, Hodge DO, et al. Role of smoking in the recurrence of atrial arrhythmias after cardioversion. Am J Cardiol. 2009; 104: 678-682.

74 Fukamizu S, Sakurada H, Takano M, et al. Effect of cigarette smoking on the risk of atrial fibrillation recurrence after pulmonary vein isolation. Journal of Arrhythmia. 2010; 26: 21-29. 